\title{
Exciting, Useful, Worrying, Futuristic: Public Perception of Artificial Intelligence in 8 Countries
}

\author{
Patrick Gage Kelley \\ Google \\ USA \\ patrickgage@acm.org \\ Christopher Moessner \\ Ipsos \\ USA \\ christopher.moessner@ipsos.com
}

\author{
Yongwei Yang \\ Google \\ USA \\ yongwei@google.com \\ Aaron Sedley \\ Google \\ USA \\ asedley@google.com
}

\author{
Courtney Heldreth \\ Google \\ USA \\ cheldreth@google.com \\ Andreas Kramm \\ Google \\ Switzerland \\ akramm@google.com
}

\author{
David T. Newman \\ Google \\ USA \\ dtnewman@google.com
}

\author{
Allison Woodruff \\ Google \\ USA \\ woodruff@acm.org
}

\begin{abstract}
As the influence and use of artificial intelligence (AI) have grown and its transformative potential has become more apparent, many questions have been raised regarding the economic, political, social, and ethical implications of its use. Public opinion plays an important role in these discussions, influencing product adoption, commercial development, research funding, and regulation. In this paper we present results of an in-depth survey of public opinion of artificial intelligence conducted with 10,005 respondents spanning eight countries and six continents. We report widespread perception that AI will have significant impact on society, accompanied by strong support for the responsible development and use of AI, and also characterize the public's sentiment towards AI with four key themes (exciting, useful, worrying, and futuristic) whose prevalence distinguishes response to $\mathrm{AI}$ in different countries.
\end{abstract}

\section{CCS CONCEPTS}

- Social and professional topics $\rightarrow$ Computing / technology policy; • Human-centered computing $\rightarrow$ Human computer interaction (HCI); • Computing methodologies $\rightarrow$ Artificial intelligence.

\section{ACM Reference Format:}

Patrick Gage Kelley, Yongwei Yang, Courtney Heldreth, Christopher Moessner, Aaron Sedley, Andreas Kramm, David T. Newman, and Allison Woodruff. 2021. Exciting, Useful, Worrying, Futuristic: Public Perception of Artificial Intelligence in 8 Countries. In Proceedings of the 2021 AAAI/ACM Conference on AI, Ethics, and Society (AIES '21), May 19-21, 2021, Virtual Event, USA. ACM, New York, NY, USA, 11 pages. https://doi.org/10.1145/3461702.3462605

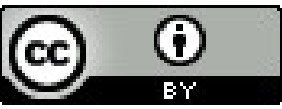

This work is licensed under a Creative Commons Attribution International 4.0 License.

AIES '21, May 19-21, 2021, Virtual Event, USA.

(C) 2021 Copyright held by the owner/author(s).

ACM ISBN 978-1-4503-8473-5/21/05.

https://doi.org/10.1145/3461702.3462605

\section{INTRODUCTION}

As the influence and use of artificial intelligence (AI) have grown and its transformative potential has become more apparent $[32,54]$, many questions have been raised regarding the economic, political, social, and ethical implications of its use [27]. The development and application of AI increasingly features in media, academic, industrial, regulatory, and public discussions [18, 23, 28], with active debate on wide-ranging issues such as the impact of automation on the future of work $[8,50,52]$, the interaction of AI with human rights issues such as privacy and discrimination $[1,4,10,16]$, the ethics of autonomous weapons [53,59], and the development and availability of dual-use technologies such as synthetic media that may be used for either benevolent or nefarious purposes [48].

Public opinion plays an important role in these discussions, influencing numerous stakeholders including advocacy groups, funding agencies, regulators, technology companies, and others [12, 14]. While there have been some explorations of public perception of AI, for example, survey research [3, 7, 13, 34, 43, 47, 59, 61], sentiment analysis [23, 26], and narrative analysis [14], much of this work has been done in Western, English-speaking contexts. Even in these better studied contexts, much remains to be learned, as both the technology and the public discussion are evolving rapidly. In this paper, we extend previous work by presenting a survey of public perception of AI conducted with 10,005 respondents spanning eight countries and six continents. Our contributions are as follows:

- We enrich current understanding of public perception by presenting results of an in-depth survey focused on AI, conducted across a broad range of countries, including several developing countries (encompassing in total: Australia, Canada, the United States (US), South Korea, France, Brazil, India, and Nigeria)

- We report widespread belief that AI will have significant impact on society, including positive expectations of AI in healthcare, as well as concerns about privacy and job loss

- We report that a plurality of participants believe that the overall impact of AI could be either positive or negative depending on 
what happens, and widespread support for responsible development and use of AI

- We identify four key sentiments (exciting, useful, worrying, and futuristic) whose prevalence distinguishes responses to AI in different countries

In the remainder of the paper, we review relevant background, describe our methodology, present and discuss our findings, and conclude.

\section{BACKGROUND}

Artificial Intelligence (AI) is a broad term with no consensus definition $[21,23,54]$, and the scope of our inquiry is intended to be similarly broad. We note that interpretation of the term is further confounded by the "AI effect" (the phenomenon that once AI successfully solves a problem and the solution becomes commonplace, it is no longer considered to be AI) [40], as well as lack of awareness of algorithmic processing in common systems [22, 49, 58]. To aid comparison with our participants' responses, following [54], we share with the reader the following definition provided by Nils J. Nilsson: "Artificial intelligence is an activity devoted to making machines intelligent, and intelligence is the quality that enables an entity to function appropriately and with foresight in its environment." [46]

\subsection{Empirical Studies}

Much of the research on public perception of AI has been surveybased, often conducted in Western, English-speaking countries such as the US and the UK [7, 13, 21, 47, 61] although this has been broadening recently. AI is often viewed as likely to have a significant impact on the future, with a frequent expectation that its effects will be positive. In a 2019 Edelman survey in the US, 9 out of 10 respondents assumed that AI will be life-changing and transformational [21]. A Gallup survey conducted in the US in 2018 found that $76 \%$ believed that AI will have a positive impact on their lives [47]; $61 \%$ of respondents had a positive view of AI and robots in a large-scale 2017 survey across Europe on the impact of digitization and automation on daily life [55]; and a 2017 consumer research survey conducted across North America, Europe, and Asia revealed a predominant expectation that society will become better $(61 \%)$ rather than worse (22\%) due to increased automation and AI [3]. A recent Pew Research survey conducted across the Americas, Europe, and Asia showed a somewhat narrower margin (possibly due to shifting public opinion, or alternatively, methodological differences), with (53\%) saying that AI has been mostly good for society versus mostly bad (22\%) [25]. Considering expected impact in the next 20 years, the 2019 World Risk Poll indicated AI would mostly help (41\%) versus mostly harm (30\%) people in one's own country, with more favorable impressions in Asia and less favorable impressions in Western countries [38, 45].

At the same time, $\mathrm{AI}$ is neither interpreted as exclusively beneficial nor exclusively disadvantageous, and public response often indicates contradictory emotions. Looking at broad reactions, Blumberg reported that US respondents were equally split between feeling optimistic and informed and feeling fearful and uninformed about AI [7], while [3] also revealed both excitement and concern. Relatedly, a 2019 Mozilla survey open to respondents on the Internet gathered continent-level demographic data and revealed varying and mixed emotions at the continent-level [43]. Specific concerns have been expressed regarding social issues, such as AI benefiting the wealthy and harming the poor, fear that AI-enabled deepfakes will erode trust in information, and $\mathrm{AI}$ increasing social isolation and reducing human capability [21]. In line with these concerns, Zhang and Dafoe found that $82 \%$ of Americans want $\mathrm{AI}$ and robots to be carefully managed [61], with $88 \%$ of Europeans expressing similar sentiment [55]. Moreover, $60 \%$ of the general population in the Edelman survey expressed the need for more regulation regarding AI development and deployment [21].

Qualitative work has also explored public perception of algorithmic systems, for example, finding that perception of algorithmic systems can vary substantially by individual factors as well as platform [17], and that end users often have fundamental questions or misconceptions about technical details of their operation $[9,22,49,57,58]$.

\subsection{Narratives and Media Sentiment Analysis}

AI is not only heavily discussed in academia, but is also a popular topic in public media [21]. 58\% of the respondents in a recent Blumberg survey indicated that they get information about AI from movies, TV, and social media [7]. In a 2016 CBS news survey, only $19 \%$ indicated not having seen any of several AI movies such as "The Terminator" or "I, Robot" [15]. Cave et al. argue that prevalent AI narratives in the English-speaking West share "a tendency towards utopian or dystopian extremes," cautioning that inaccurate narratives could affect technological advancement and regulation [14], with similar points raised in [32, 54,60]. Cave et al. surveyed UK respondents regarding their responses to eight dominant narratives about AI, reporting that the strong majority elicited more concern than excitement [13]. At the same time, sentiment analysis of newspaper articles from the New York Times and associated content found that, in general, AI has had consistently more optimistic than pessimistic coverage over time [23], and did not support the hypothesis that news media coverage of $\mathrm{AI}$ is negative [26].

\subsection{National Considerations}

A number of countries have established national strategies to promote the use and development of AI, which vary by country and may influence public perception [20]. ${ }^{1}$ The importance of studying local context is also illustrated by analysis of country-specific opportunities and challenges for AI, e.g. [36]. Further, researchers have called for better integration of developing country considerations in the discussion and development of AI [51].

\section{METHODOLOGY}

In order to better understand public perception of AI, we partnered with Ipsos, a global market research firm, to conduct a survey of 10,005 respondents in eight countries in July 2019. Methodologically, this work falls in the genre of public opinion polling, as described below.

\footnotetext{
${ }^{1}$ See also https://futureoflife.org/national-international-ai-strategies/
} 


\subsection{Instrument Development and Translation}

To develop concepts and questions, we consulted experts at our institutions, reviewed published work, drew on our own previous unpublished research, and conducted an initial pilot survey in June 2018 with 1300 respondents drawn from a panel of the general online population in the US. Many questions in the final instrument ${ }^{2}$ were written uniquely for this survey while others were modified from or replicate other questions in the literature or the canon of public opinion surveys. In order to more accurately reflect real-world settings, we did not define AI, and left interpretation of the term to the respondents. ${ }^{3}$ We included primarily closed-form questions as well as a few open-ended questions for free responses. We also included standard demographic questions such as age, gender, education, income, region, and urbanicity. The final instrument included several dozen questions on a range of topics related to artificial intelligence. In this paper we focus on select questions related to the research objectives below.

Recognizing that public response and adoption are informed by intertwined cognitive and emotional factors [31,37], we explore the following research objectives (the first being cognitive, the second being emotional, and the third considering how they vary by country):

- What social impact do respondents anticipate AI will have?

- How do respondents feel about AI?

- How do the issues above vary by country?

After completing the instrument in English, we engaged cApStAn, a linguistic quality assurance agency with expertise in survey translation. We made several improvements based on their insights to minimize terminology that would be difficult to translate. In consultation with cApStAn, we also developed a translation style guide and question-by-question translation guidelines for each target language, for example, specifying translations of key terms and standard scales for Likert questions in each language to ensure consistency (see Table 1 for the languages we offered).

Our market research partner's in-country translation teams and/or third party vendors then translated the full instrument to all target languages while referring to the style guides and guidelines. When the instrument was fully translated, it was provided to us for final review, and the translations were revised through an iterative process before final sign-off and deployment. After the survey was complete, a professional translation vendor provided verbatim translations for all non-English responses; these verbatim translations are used in example quotes in this paper.

\subsection{Deployment}

We selected a range of countries with different characteristics, such as stage of technological development, nature of the workforce, and varied development indices. The survey was fielded to online panels (groups of respondents who have agreed to participate in surveys over a period of time) representative of the online population in each country. Consistent with the best panels available for online market research, such panels tend to be broadly representative of

\footnotetext{
${ }^{2}$ For the questions used in the instrument see: arXiv:2001.00081

${ }^{3}$ We note that in our pilot, we had two versions of the survey (one that defined $\mathrm{AI}$ and one that did not) and responses to subsequent questions were similar regardless of whether a definition had been provided.
}

the general population in countries with high access to technology, but less representative of the general population in countries with more limited access to technology; for example, in developing countries they tend to skew urban. Respondents were recruited using stratified sampling (a method of recruiting specific numbers of participants within demographic subgroups), with hard quotas on age and gender in each country. A summary of countries and demographics is provided in Table 1.

The median survey length was 23 minutes across all completions, including those who said they had never heard of AI in an early screening question and received a much shorter variant of the survey (see Appendix). All respondents received incentives in a point system or cash at an industry-standard amount for their market.

\subsection{Data Processing and Analysis}

3.3.1 Quality Checks. The market research firm conducted quantitative and qualitative checks to remove low quality responses on an ongoing basis until the quota was reached in each country. Example grounds for removal included being identified as a bot, speeding (answering substantially more quickly than the median time), or providing nonsensical or profane responses to open-ended questions. Overall we removed $7.3 \%$ of responses for quality. After data collection was complete, standard procedures were followed to apply a modest weighting adjustment to each respondent so that the samples in each country are more representative [6]. The variables considered in weighting appear in Table 1.

3.3.2 Coding of Open-Ended Responses. We reviewed the openended responses from the pilot to identify emergent themes [5] and develop an initial codebook for all questions, then iterated as we reviewed responses from all countries to refine it as necessary. The open-ended responses were coded in the native language by our market research partner's dedicated coding team (English and French) and one of their third party coding vendors (all other languages). As described in McDonald et al., a variety of different approaches may be employed to improve the reliability of qualitative analysis [41]. In our case, following best practices in public opinion research for coding against multiple languages, we used professional coders, followed an iterative process to continuously improve the codes, and performed a series of hierarchical quality checks. While coders were specialized by language, they worked together to ensure consistency, sharing notes in specialized coding software. Both we and our market research partner performed multiple levels of quality checks on the resulting coding, randomly sampling from all responses in each country as well as checking all instances of select codes.

For the open-ended question regarding the feelings or emotions the respondent associated with AI, we began by following the process described above; the resulting codebook for this question encompassed 92 codes (e.g. 'Useful,' 'Skeptical,' 'AI takes over') and specified that multiple codes could be assigned per response. After these codes were assigned and we reviewed the open-ended verbatim responses in detail, four groups of codes emerged from the data as common and semantically distinct (Exciting, Useful, Worrying, and Futuristic) - for example, the Useful group encompassed codes such as 'Useful,' 'Helpful,' 'Productivity,' etc. We 


\begin{tabular}{|c|c|c|c|c|c|c|c|c|}
\hline Country & AUS & CA & US & KR & FR & BR & IN & NG \\
\hline HDI Rank & $8^{\text {th }}$ & $16^{\text {th }}$ & $17^{\text {th }}$ & $23^{\mathrm{rd}}$ & $26^{\text {th }}$ & $84^{\text {th }}$ & $131^{\text {st }}$ & $161^{\text {st }}$ \\
\hline $\begin{array}{l}\text { Languages } \\
\text { offered }\end{array}$ & English & $\begin{array}{l}\text { English, } \\
\text { French }\end{array}$ & English & Korean & French & $\begin{array}{l}\text { Brazilian } \\
\text { Portuguese }\end{array}$ & $\begin{array}{l}\text { English, } \\
\text { Hindi }\end{array}$ & English \\
\hline Weighting & $\begin{array}{l}\text { age, gender, } \\
\text { education, } \\
\text { region }\end{array}$ & $\begin{array}{l}\text { age, gender, } \\
\text { education, } \\
\text { region }\end{array}$ & $\begin{array}{l}\text { age, gender, } \\
\text { education, } \\
\text { region, race }\end{array}$ & $\begin{array}{l}\text { age, gender, } \\
\text { education, } \\
\text { region }\end{array}$ & $\begin{array}{l}\text { age, gender, } \\
\text { education, } \\
\text { region }\end{array}$ & $\begin{array}{l}\text { age, gender, } \\
\text { education, } \\
\text { region }\end{array}$ & $\begin{array}{l}\text { age, gender, } \\
\text { education }\end{array}$ & $\begin{array}{l}\text { age, gender, } \\
\text { education }\end{array}$ \\
\hline Respondents & 1000 & 1500 & 1501 & 1000 & 1001 & 1503 & 1500 & 1000 \\
\hline Age & $\begin{array}{l}M=43 \\
S D=15.3\end{array}$ & $\begin{array}{l}M=44 \\
S D=16.0\end{array}$ & $\begin{array}{l}M=44 \\
S D=17.5\end{array}$ & $\begin{array}{l}M=40 \\
S D=12.4\end{array}$ & $\begin{array}{l}M=43 \\
S D=15.4\end{array}$ & $\begin{array}{l}M=34 \\
S D=12.3\end{array}$ & $\begin{array}{l}M=30 \\
S D=8.9\end{array}$ & $\begin{array}{l}M=31 \\
S D=9.0\end{array}$ \\
\hline Gender & $\begin{array}{l}49 \% \text { male } \\
51 \% \text { female }\end{array}$ & $\begin{array}{l}47 \% \text { male } \\
53 \% \text { female }\end{array}$ & $\begin{array}{l}49 \% \text { male } \\
51 \% \text { female }\end{array}$ & $\begin{array}{l}52 \% \text { male } \\
48 \% \text { female }\end{array}$ & $\begin{array}{l}50 \% \text { male } \\
50 \% \text { female }\end{array}$ & $\begin{array}{l}49 \% \text { male } \\
51 \% \text { female }\end{array}$ & $\begin{array}{l}70 \% \text { male } \\
30 \% \text { female }\end{array}$ & $\begin{array}{l}63 \% \text { male } \\
37 \% \text { female }\end{array}$ \\
\hline
\end{tabular}

Table 1: Country details, respondent summary and demographics.

assigned each of the 92 codes to exactly one of these four groups or Other accordingly. Other encompassed answers that were inarticulate, classified as unable to be coded, mentions of technology without any sentiment (e.g. "computer" or "technology"), and a long tail of other opinions on AI (for example "curiosity" or "surprise"). Based on the codes that each response had been assigned, each response was considered to be part of those group(s) - for example, if a response had been assigned the code 'Helpful' and the code 'Concern,' that response was part of the sentiment groups Useful and Worrying. A response that received only codes labeled Other appears in None.

When we report statistical results for open-ended questions below (for example, the frequency of a code for a given question), these results apply only to the specific question. When we present illustrative quotes, we often draw responses from across any of our four open-ended questions, as relevant responses and similar coding often applied across them.

3.3.3 Analysis. We used an inductive approach which involved exploring emerging themes and common patterns in the data [29]. When participants qualified to take the survey, they were required to provide an answer to each question, though many questions offered an option for the respondent to indicate they did not know the answer. All respondents were shown three questions and select additional questions were filtered if participants had never heard of AI. In this report, we designate participants who had at least heard of AI as 'AI-aware.'

As the impact and use of AI expands worldwide, how people learn about, interact with, and use AI varies. People from developed countries (i.e. countries that are more industrialized and have higher per capita incomes, which include Australia, Canada, the US, South Korea, and France) have different needs than people from developing countries (i.e. countries that are less industrialized and have lower per capita incomes, which include Brazil, India, and Nigeria), and this shapes how AI is perceived, adopted, and normalized globally $[51,56]$. Therefore, we anticipated that there might be meaningful differences in AI perceptions associated with development level. We include the Human Development Index (HDI) Rank in Table $1 .{ }^{4}$

\subsection{Limitations}

We note several limitations of our methodology that should be considered when interpreting this work. First, it carries with it the standard issues attendant with survey methodology, such as the risk of respondents misunderstanding questions, poor quality translation, or respondents satisficing [30] or plagiarizing open-ended responses. We have worked to minimize these risks through piloting, use of open-ended questions in conjunction with closed-form questions, translation style guides and review, and data quality checks. We also note that panels in India are well-known in the industry to be disproportionately likely to have a social desirability response bias (as defined in [30]), so optimism in the Indian responses should be considered in that context. Second, online panels are not representative of the general population. While we have used a high standard of currently available online panels, we caveat our findings as not representative of the general population, particularly in Brazil, India, and Nigeria. Third, while members of the research team and/or market research partner team have experience conducting research in all markets studied, members of the team reside in Western countries. We have worked to minimize the risk of misinterpretation by collaboration and discussion with in-country partner teams but recognize that our interpretations may lack context or nuance that would have been more readily available to local residents.

\section{FINDINGS}

In this section, we first provide contextualizing information about how respondents describe AI, and how they report learning about it. We then turn to respondents' expectations of the impact of AI on society. We conclude this section by exploring respondents' sentiment regarding AI.

\footnotetext{
${ }^{4}$ We show HDI ranks from the 2020 Human Development Report http://hdr.undp.org/ en/content/developing-regions, which uses HDI values from 2019, aligning with the dates of our survey deployment.
} 


\subsection{Description and Exposure}

We begin with brief context on what respondents understand AI to be. In response to an open-ended question in which we asked respondents to describe AI, respondents across countries often mentioned concepts like computers or robots that think independently, learn, or perform human tasks.

a machine that can think for itself - Australia $^{5}$

Extremely calculative robot -Nigeria

Artificial Intelligence, to my understanding, is the programming of computers in order for them to learn using different experiences and through lots of different examples. -Canada

It is a robot or device that is made to be able to preform task as good as if not better than a human -US

Respondents' comments sometimes indicated partial knowledge, associated AI with other technologies such as the Internet or the Internet of Things (IoT), described AI as something non-technological, or indicated that they did not know.

Something I do not understand, but which appears to be a major technological revolution. - Brazil

Only thing that I can think of is chess playing programs. However, I'm sure there are more applications in use - technology companies must be using AI somehow. -Australia

AI is used in many ways which a ordinary person like me won't even be able to think off. -India

AI is a type of technology that makes all your gadget or that connects all your tech stuff all together to make life more easier -Nigeria

Respondents reported learning about $\mathrm{AI}$ in many places, the top five being: social media (45\%), TV reports and commentaries $(42 \%)$, movies or TV shows (40\%), magazine or online articles $(32 \%)$, and family and friends (31\%). While not in the top five, personal experience using products that have AI technology also drives awareness (13\%). The examples below illustrate media influence:

A recent bad example with the 737 Max aircraft that made decisions on their own, resulting in two accidents with many victims. -Brazil

I am intrigued by this idea. Computers being able to write a screen play in 15 minutes is amazing. I am a writer and just heard a pod cast about this. $-U S$

Does no one watch movies, read, or anything to do with science fiction!!! It ALWAYS ends badly... there is just no good outcome, that I can see (for now at least), to an actual, fully fledged, AI. $-U S$

I have recently heard in news and everywhere $\mathrm{AI}$ is now the latest invention the man can acheive -India

\subsection{Widespread Expectation That AI Will Change The World}

In this section, we present respondents' expectations of the impacts of AI on society. We begin by discussing respondents' strong expectation that AI will have significant impact, and then discuss some of the specific domains in which they expect that impact to occur. We then discuss the valence of their expectations, whether

\footnotetext{
${ }^{5}$ We use verbatim responses throughout (in some cases translated) and do not correct typographic or grammatical errors.
}

positive, negative, or ambivalent. We close this section by drawing together these ideas with respondents' strong desire for responsible innovation and care in the development and deployment of AI.

Many of our respondents believe that AI will be transformative. Across all eight countries, only a tiny number $(1-2 \%, M=1 \%)$ indicate that AI "won't have much effect on society" in the long term (see Table 2). Similar sentiment can be observed in the openended responses:
AI will revolutionise the way we live in our future. -India
New and improved tech that will change our lives for good -Nigeria
we are entering a new era. Very modern -Canada

Machines taking over humans!! :) on a serious note, A.I. is making things possible we thought were not possible a few years ago. Computers recognise faces and fingerprints of humans. Machines carry out so many things to assist humans. Everywhere we look there are examples of artificial intelligence around us. -Australia

It makes me think about how this is going to shape our future and I feel excited by that. -Australia

We see this reflected in specific aspects of life as well, for example, with low levels of people reporting that AI will have "no change" on measures such as healthcare $(9-27 \%, M=19 \%)$, privacy $(6-25 \%$, $M=15 \%)$, and jobs $(7-21 \%, M=15 \%)$ in the future. ${ }^{6}$ Across all countries surveyed, AI was seen as promising for healthcare. Most respondents believe AI will lead to "better healthcare" in the future ( $M=56 \%$ "better" versus $M=12 \%$ "worse" across all countries), with Nigeria the highest with $78 \%$ (positive-to-negative ratio = 19.5) expecting improvement. This optimism resonates with media narratives encouraging AI adoption in healthcare efforts. ${ }^{7,8}$

Progress, I know it will impact positively especially in the areas of health care. -Nigeria

I believe it has great opportunities for advancement in medical technology. It has moderate use in banking. It has the ability to provide useful information instantaneously. It will greatly improve the quality of mundane tasks. -Canada

Expectations of AI-related change are not always positive, with concerns about privacy, job loss, and harm to personal relationships in all countries. Respondents saw privacy concerns as a likely downside of AI, with a plurality of respondents in all countries except for India (50-64\% in other countries; $31 \%$ in India) believing they will have less privacy in the future because of $\mathrm{AI}(M=52 \%$ "less" versus $M=23 \%$ "more" across all countries).

A new frontier. Very exciting and scary at the same time. Lots to gain but will personal privacy be the price? -Australia

optimistic that it will enhance peoples lives and bring about breakthroughs in many fields but also skeptical that people will lose their jobs and there will be an invasion of privacy -Canada

A trending mobile app that undresses people. It violates privacy rules -Nigeria

\footnotetext{
${ }^{6}$ These ranges are the spread across the eight countries, and $M$ is the response across our entire sample. For example, for privacy: $6 \%$ of respondents thought there would be no change in Nigeria, 25\% thought there would be no change in France, and 15\% across all respondents.

${ }^{7}$ https://borgenproject.org/ai-in-african-healthcare-revolutionizing-the-industry

${ }^{8}$ https://allafrica.com/stories/201907040526.html
} 


\begin{tabular}{lrrrrrrrr} 
Country & AU & CA & US & KR & FR & BR & IN & NG \\
\hline Respondents (n) AI-aware & 946 & 1424 & 1406 & 995 & 970 & 1481 & 1472 & 967
\end{tabular}

Overall, in the long term, Artificial Intelligence (AI) will be...

Mostly bad for society

Either good or bad for society, depending on what happens

Good and bad in roughly even amounts

Won't have much effect on society

Don't know
Mostly good for society

\begin{tabular}{rrrrrrrr}
$18 \%$ & $20 \%$ & $21 \%$ & $23 \%$ & $18 \%$ & $38 \%$ & $51 \%$ & $37 \%$ \\
$14 \%$ & $15 \%$ & $17 \%$ & $8 \%$ & $14 \%$ & $7 \%$ & $8 \%$ & $4 \%$ \\
$43 \%$ & $39 \%$ & $40 \%$ & $60 \%$ & $42 \%$ & $41 \%$ & $26 \%$ & $48 \%$ \\
$14 \%$ & $17 \%$ & $13 \%$ & $6 \%$ & $13 \%$ & $10 \%$ & $12 \%$ & $9 \%$ \\
$1 \%$ & $1 \%$ & $1 \%$ & $1 \%$ & $2 \%$ & $1 \%$ & $1 \%$ & $1 \%$ \\
$9 \%$ & $8 \%$ & $8 \%$ & $2 \%$ & $11 \%$ & $4 \%$ & $2 \%$ & $2 \%$ \\
\hline
\end{tabular}

Table 2: Public opinion regarding the long-term impact on society from respondents who reported being aware of AI.

\section{IT DICTATED MY WEIGHT AND HEIGHT IN PUBLIC -India}

Ads that show up on computers after visiting websites is one thing, but ads that show up after just talking about something makes me think my phone is listening in on my conversations $-U S$

Respondents expected that AI will heavily impact the number of jobs available in the future. Across all countries surveyed, many respondents (39-76\%, $M=53 \%$ ) expected that AI will contribute to "more jobs lost" in the future, while far fewer expected it to lead to "more jobs created" $(7-43 \%, M=22 \%)$. The open-ended data further emphasizes such concerns, illustrating the perception that AI may replace humans or make them less necessary in the workforce, as well as the association of robots in particular with job loss (due to their ability to perform human tasks), and in rare cases personal experiences related to job loss:

I feel that it has taken away jobs $-U S$

A highly computerised potentially dangerous job stealing system of machinery operation -Australia

New technologies. Convenience in life. Reduction in jobs. -South Korea

Am happy about it but am still sceptical about it. This is because it might probably put some persons out of work -Nigeria

Unemployment comes to my mind when I hear the phrase Artificial Intelligence(AI). - India

Respondents also anticipate that AI will impact personal relationships in the future. South Koreans expressed the highest concern that AI will weaken personal relationships, with $61 \%$ of respondents saying so (34-50\% "weaker" across the other countries, all: $M=46 \%$, versus $9-45 \%$ "stronger", $M=22 \%$ ), resonating with expectations expressed in the open-ended responses that $\mathrm{AI}$ and robots will replace humans.

fear that during my lifetime I will be interacting more with AI than live humans -US

It helps the future by making things easier, but diminishes employment and human contact. -France

Makes life convenient and can replace humans -South Korea

Beyond these specific expectations for sector-based change in healthcare, jobs, privacy, and personal relationships, across all our respondents $29 \%$ expected AI would be mostly good for society overall. There were clear differences between countries, and by considering HDI rank, we see developing countries taking a more optimistic view of its future effects. Respondents in Brazil, India, and Nigeria reported AI would be mostly good for society (37-51\%) while in Australia, Canada, the US, South Korea, and France that range was lower (18-23\%). Nonetheless, ambivalence was evident in all eight countries. Across all countries, $41 \%$ of respondents believe that in the long term AI will be either good or bad for society, depending on what happens, and $12 \%$ believe it will be good and bad in roughly even amounts. Respondents sometimes shared mixed emotions in open-ended responses as well:

It is a wonderful and terrifying concept that is inevitable. -Australia

A little excitement and a little terror $-U S$

The future of our world in a way that represents both progress and destruction -Canada

Respondents further highlighted that the effects of AI could be either positive or negative, or both positive and negative:

Mixture of amazement at the potential of this technology and concern about possible pitfalls. Could be the start of something amazing or the beginning of the end (a la Terminator).

-Australia

A mixture of knowledge and fear. I know that it will help or is already helping in several important areas, but there is always that fear that one of these AIs will become too autonomous and turn against us. - Brazil

Life will be much more enjoyable, but I fear that we'd lost what makes us human. Robots will replace humans in various fields, but there are positive sides as well, a pet robot being one of them. - South Korea

Artificial intelligence is something most people will come to depend on in a few decades. It will make life easier at the same time make people lose their jobs. But one I'm certain of is that $\mathrm{AI}$ is here to stay for good. -Nigeria

It can help us a great deal in the future if it is used for the good of humanity, but we also run the risk of all this software generating major chaos! -Brazil

Unsure about the net value - has lots of positives but also there are some very legitimate concerns. -Canada

It's exciting to think about the things that could come about with AI that would make our lives easier and safer, but also scary of course, who knows how it will truly effect society -US

This potential for AI to have variable effects was reflected in all eight countries in response to questions about the importance 
of large technology companies following responsible innovation practices. $82 \%$ of respondents thought it was very or extremely important for these companies to "Carefully weigh the pros and cons before releasing new technologies" and similarly $81 \%$ thought they should "Follow a responsible process to develop products that use AI." These strong levels of support illustrate the high value respondents place on responsible development and use of AI. Openended responses also revealed respondents' expectation that the effects of AI depend on whether it is used responsibly.

Artificial intelligence worries me a bit because if it's not used well it can be dangerous, it has no conscience or ethics, but I acknowledge that it is an amazing tool. -France

Depends in the context. Customer service ai? Great. Ai drones very bad -Australia

A bit excited because it makes job quite easy but again its scary if it the technology goes wrong like someone using it for evil purposes. -Nigeria

Angry that future concerns or negative impacts aren't ever considered before technology is developed -Australia

Artificial Intelligence is very useful for whole human world. But don't use it in a bad way -India

\subsection{Sentiment Groups}

Respondent sentiment towards AI was concordant with the results presented in the previous sections, with respondents demonstrating emotions reflective of AI's potential impact. In this subsection we describe the sentiment groups that emerged from our analysis and explore their variation across countries.

Responses to the open-ended question 'What feelings or emotions come to mind when you hear the phrase Artificial Intelligence (AI)?' were assigned to sentiment groups as described in the methodology section. Multiple assignments were possible, so a response such as "fear and excited at the same time" (US Respondent) would be included in Worrying and Exciting, but not Useful or Futuristic. See Figure 1.

- Exciting $(18.9 \%, N=1888)$ - responses in this group contained positive feelings about AI and often exhibited broad excitement or enthusiasm.

- Useful (12.2\%, $N=1223)$ - responses in this group expressed the belief that AI will be helpful and assist humans in completing tasks.

- Worrying $(22.7 \%, N=2269)$ - unlike the previous two groups, which each capture a relatively tight set of responses to $\mathrm{AI}$ in our open-ended data, this category comprises a wide range of negative emotional responses, predominantly various forms of concern and fear

- Futuristic $(24.4 \%, N=2439)$ - responses in this group are not necessarily positive or negative towards $\mathrm{AI},{ }^{9}$ but rather are included for any mention of the futuristic nature of AI, whether by simply describing AI as advanced; mentioning robots, aliens, or other science-fiction concepts; or by referencing the future directly.

\footnotetext{
${ }^{9}$ Although Futuristic may not traditionally be seen as a sentiment, when we asked participants to describe their feelings or emotions about AI, they organically responded at very high rates (19\% to $38 \%$ across the countries).
}

The groups above do not cover all responses. About a third of our sample $(34.9 \%, N=3489)$ fell into the None group described in the methodology section. This $34.9 \%$ of responses were assigned only the 48 codes that we did not include in the groups. See Table 3.

We now turn to country-level observations, where we see strikingly different national patterns in response towards AI across the eight countries we studied. Four countries most often find AI Worrying; three countries find AI to be predominantly Exciting; and only our South Korean respondents are most likely to discuss how Useful AI can be. We visually represent the character of these differences in Figure 1.

Consistent with our expectation that developed countries (those most-developed, by HDI rank) would share similarities, the dominant sentiment group in Australia, Canada, and the US was Worrying, followed by Futuristic (see Table 3). This resonates with claims that popular press and media narratives in these regions have emphasized potential threats of AI [14, 23, 32]. France shares characteristics with Australia, Canada, and the US, with similar values for the Useful, Worrying and Futuristic groups, but with fewer in the Exciting group.

South Korea has a unique profile among the countries surveyed, having the largest percentage in the both the Useful $(19 \%, N=192)$ and Futuristic (38\%, $N=379)$ sentiment groups. South Korean respondents also had the lowest percentage of Exciting ( $6 \%, N=63$ vs $10-37 \%$ in all other countries). These findings are consistent with South Koreans' high level of exposure to technology: South Korea boasts the world's highest robot density [33], is one of the largest global investors in smart buildings [19], and may be "at the vanguard of a revolution in AI and big data healthcare" [44]. Consistent with this, South Korean respondents often mentioned $\mathrm{AI}$ assistants and home automation, which may contextualize AI as a more familiar, everyday technology:

\section{AI is everywhere from hospitals to homes and cars. -South Korea \\ Use big data to make daily life more convenient. -South Korea \\ Convenient, relaxed daily life -South Korea \\ With just the smartphone, I can check the gas, temperature, and the foods in the fridge. -South Korea \\ Self-driving car, automated production, convenient daily life -South Korea}

In the developing countries (those less-developed by HDI rank, i.e., Brazil, India, and Nigeria), Exciting was the dominant sentiment, as seen in Figure 1/Table 3. Brazil also has a unique profile among the countries surveyed, with higher levels of Worrying and Futuristic relative to the other developing countries. However, Brazilian respondents reported levels of Exciting and Useful similar to those in the other developing countries. India was the most enthusiastic about AI, with the highest level for Exciting, the second highest level for Useful, and the lowest level for Worrying across all countries.

We now consider the relationship between sentiment towards $\mathrm{AI}$ and expected impact on society. In Figure 2, we show how the respondents we included in each of our four sentiment groups reported believing AI would impact society. For example, of the 1802 respondents who expressed the sentiment Exciting, $49 \%$ of 

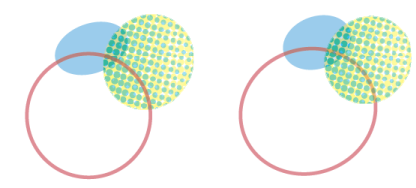

Australia

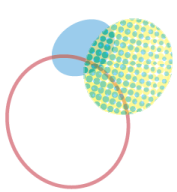

United States

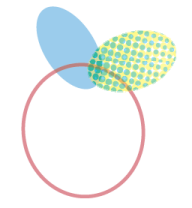

France

\section{Useful}

Useful, Helpful, Productivity, Benefits, Assistance,

Human tasks

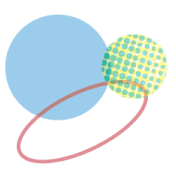

South Korea

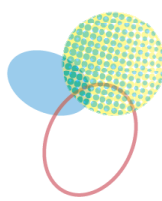

Brazil

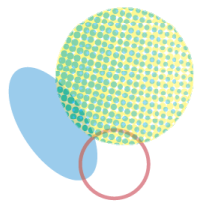

India

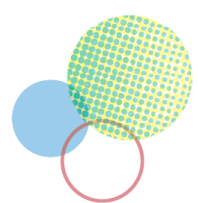

Nigeria
FE⿱

\section{Exciting}

Excited, I like it, Good, Hope Happy, Blessed, Amazing

\section{Worrying}

Concern, Cautious, Skeptical, Creepy, Danger, Distrust, Fear, Hurt, Bad, I don't like it

Privacy, Always listening, Surveillance, Tracking, Other privacy terms

Al replaces humans, Al takes over, Humans get lazy, Humans get less skilled, Job loss, Too advanced

It is interesting and useful, but I am worried about lost jobs, not to mention AI getting smart enough to take over and control us. -Canada

A little bit of fear because I don't know the limit of Artificial intelligence (if there is a limit) -Nigeria

This topic is thought-provoking. It generates fear and also curiosity and concern. -Brazil

Progress but danger. Fear, uncertainty. -France

Fearful of our future robot overlords -Australia
Artificial intelligence is the future. It will bring the dawn of a new age -Nigeria

$\mathrm{AI}$ is the new trend for technology, I myself being a tech geek i know that $\mathrm{AI}$ is soon going to change the whole world with it's endless possibilities. AI is the future of Mankind -India

Advanced technology -France

I try to make an effort to follow this futuristic trend. I really like it and I am onboard with AI in general sense. -Brazil

its magnificient technology of tomorrow -India

Figure 1: Description of our four sentiment groups, with the complete list of codes that comprises each, and example responses. While we use the responses to illustrate a particular sentiment, some of them fall in multiple sentiment groups, as sometimes occurred in our data set. At the top of the figure, we represent the overlap between the groups with Venn diagrams, using 3-Venn diagrams which exclude Futuristic for readability. The alert reader may wonder why we use oblong circles; these more accurately represent the area in the overlap. We use the method and tooling described in [42]. As throughout the paper we order by HDI, with the exception of South Korea and France, to highlight how similar France is to Australia, Canada, and the United States.

\begin{tabular}{lrrrrrrrr} 
Country & AU & CA & US & KR & FR & BR & IN & NG \\
\hline Respondents $(n)$ All & 1000 & 1500 & 1501 & 1000 & 1001 & 1503 & 1500 & 1000 \\
\hline Exciting & $17 \%$ & $14 \%$ & $15 \%$ & $6 \%$ & $10 \%$ & $23 \%$ & $36 \%$ & $25 \%$ \\
Useful & $9 \%$ & $9 \%$ & $7 \%$ & $19 \%$ & $11 \%$ & $14 \%$ & $18 \%$ & $11 \%$ \\
Worrying & $31 \%$ & $33 \%$ & $30 \%$ & $14 \%$ & $31 \%$ & $21 \%$ & $9 \%$ & $11 \%$ \\
Futuristic & $22 \%$ & $21 \%$ & $19 \%$ & $38 \%$ & $20 \%$ & $34 \%$ & $24 \%$ & $19 \%$ \\
None & $38 \%$ & $39 \%$ & $42 \%$ & $31 \%$ & $39 \%$ & $25 \%$ & $27 \%$ & $41 \%$
\end{tabular}

Table 3: Percentage of respondents from each country whose open-ended sentiment was coded to be in one of our groups. Respondents can appear in multiple sentiment groups, however a respondent whose answers received only codes not in these groups appears in None. 


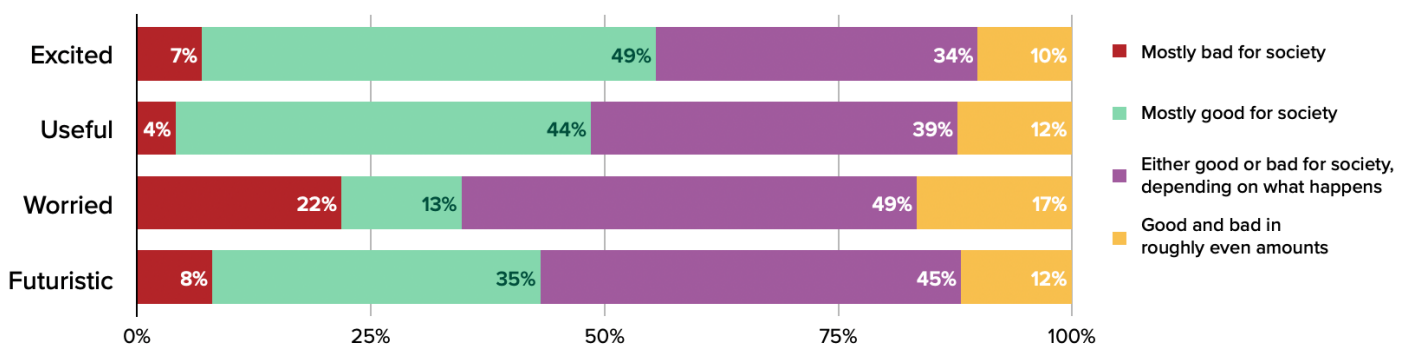

Figure 2: For each sentiment, we show all respondents who were coded as having that sentiment, grouped by their answer to how AI would impact society.

them said AI would be mostly good for society, while only $13 \%$ of the 2129 respondents who expressed Worrying thought this. While we do see some overlap between the expectations of AI and our sentiment groups, the sentiments we observed do not fully account for responses regarding societal impact, and vice versa. This provides further support for measuring public opinion of both cognitive and emotional factors regarding AI, as well as laying the groundwork for further investigation of what drives these factors as we discuss further below.

\section{DISCUSSION}

Based on our respondents views of both how they expect AI will transform society and how they feel about AI, we argue for three areas of continued investment: design and ethics resources to help more responsibly build the systems that the public believes will shape the future; additional investment and research into information campaigns to educate about AI, as well as increased design and development of offerings the public values; and further research into the media narratives that are shaping public expectations and sentiment, particularly analysis that explores the nuanced space beyond positive versus negative valence.

\subsection{AI Design and Ethics Guidance}

Our results on public interest in the transformative nature of AI and its responsible use underscore the importance of the growing body of AI design guidance and ethical toolkits. Such resources provide practical strategies to responsibly build and deploy systems which the public expects will matter so much to society, and our findings argue for their increased prioritization and application.

AI designers and developers can leverage work on the relationship between AI and HCI (under the names Human-Centered Machine Learning [39], Machine Learning UX or MLUX [11], and similar terms), such as Amershi et al.'s synthesis of twenty years of $\mathrm{AI}$ design learnings into 18 guidelines for human-AI interaction design [2], the People + AI Guidebook, ${ }^{10}$ and other resources outlined in Carney's summary [11].

Regarding ethical resources, our findings reinforce the importance of designing and developing AI responsibly to benefit society and minimize potential harms [1,28], and of sharing information about those efforts. Recent analyses summarize the rapidly increasing number of principles and guidelines for ethical AI [24,35], and

\footnotetext{
${ }^{10}$ https://pair.withgoogle.com/
}

tactical support for applying these ideas in practice is available in resources such as the Markkula Center Ethics in Technology Practice Framework and Toolkit, ${ }^{11}$ the Omidyar Ethical OS Toolkit, ${ }^{12}$ and the Princeton Dialogues on AI and Ethics Case Studies. ${ }^{13}$

\subsection{Interventions and Communications}

Public misperception or unrealistic expectations of AI can lead to unfounded fears or disappointment and disillusionment [7, 14]. Our insights into public opinion, particularly in countries where little data has been gathered previously, suggest areas in which the public may benefit from additional information and educational programs. They also suggest ways in which the design and development of particular technologies may have a favorable impact on public opinion. For example, future research could explore the conditions facilitating South Korea's unusually strong impression of AI as Useful, to gain insight into whether or how this sentiment might resonate elsewhere via communications or technological offerings. As another example, our findings point to the value of emphasizing AI's application to healthcare in communications as well as product and research investments. Finally, many respondents were concerned about negative impacts of AI on privacy, reinforcing the value of continued emphasis on designing and developing AI with privacy in mind, concordant with discussion of privacy by design in the EU General Data Protection Regulation (GDPR). ${ }^{14}$ The privacy discussion continues to evolve quickly, and best practices for AI technologies continue to be actively explored in the academic, legal, and policy communities, offering many opportunities for advances in this area.

\subsection{Narratives and Sentiment Groups}

Our results reflect a number of key dialogues that have appeared in public discussion and the media, for example, that AI offers significant improvements for health; that $\mathrm{AI}$ is associated with privacy issues, job loss, and social isolation; and that AI could be either a significant boon or a significant threat to humanity. Other work examining narratives in Western, English-speaking countries has argued that popular portrayals of AI exaggerate this dichotomy [14]. Our findings suggest that concern about AI is

\footnotetext{
${ }^{1}$ https://www.scu.edu/ethics-in-technology-practice/

${ }^{12} \mathrm{https}: / /$ ethicalos.org/

${ }^{13} \mathrm{https} / / /$ aiethics.princeton.edu/case-studies/

${ }^{14}$ https://eugdpr.org/
} 
higher in these countries as well as Australia and France, but is less prominent in South Korea, Brazil, India, and Nigeria.

More broadly, our findings revealed sentiment groups as a distinguishing feature, with respondents in different countries finding AI to be Exciting, Useful, Worrying, and Futuristic to varying degrees. These groups provide one nuanced alternative to understanding people's feelings towards AI, rather than considering their orientation to AI as simply positive or negative. Now that these differences between countries have been observed, it would be valuable to learn more about what drives them, e.g. to formally measure and analyze the relationship between media and pop culture narratives in different countries and the presence of these sentiment groups, as well as tracing the relationship and movement of narratives across countries.

Further, it would be useful to explore other factors that likely influence these sentiment groups, such as country culture and economy; presence, awareness, and availability of AI technologies such as customer service chatbots, personal assistants, and more; and personal, formative experiences using AI technology. It would also be worthwhile to explore how sentiment groups affect behavior such as adoption of AI technologies and public opinion on topics such as research funding and regulation.

\section{CONCLUSIONS}

We surveyed public opinion of artificial intelligence with 10,005 respondents spanning eight countries across six continents, focusing on issues such as expected impacts of AI, sentiment towards AI, and variation in response by country. We report widespread perception that AI will have significant impact on society but the overall nature of these effects is not yet determined, underscoring the importance of responsible development and use. We identify four groups of sentiment towards AI (Exciting, Useful, Worrying, and Futuristic) whose prevalence distinguishes different countries' perception of AI. Our findings suggest opportunities for future work, such as empirical study of the relationship between media narratives and sentiment across countries, and opportunities for interventions and communications regarding the design and development of AI technologies.

\section{ACKNOWLEDGMENTS}

We thank Elie Bursztein, Ed Chi, Charina Chou, Jen Gennai, Jake Lucchi, Ken Rubinstein, and Kurt Thomas for valuable contributions to this work. We also thank the cApStAn team for their important contributions to linguistic quality and the Ipsos team for their excellent work fielding the survey.

\section{REFERENCES}

[1] Christophe Abrassart, Yoshua Bengio, Guillaume Chicoisne, Nathalie de MarcellisWarin, Marc-Antoine Dilhac, Sebastien Gambs, Vincent Gautrais, Martin Gibert, Lyse Langlois, Francois Laviolette, Pascale Lehoux, Jocelyn Maclure, Marie Martel, Joelle Pineau, Peter Railton, Catherine Regis, Christine Tappolet, and Nathalie Voarino. 2018. Montreal Declaration for Responsible Development of Artificial Intelligence.

[2] Saleema Amershi, Dan Weld, Mihaela Vorvoreanu, Adam Fourney, Besmira Nushi, Penny Collisson, Jina Suh, Shamsi Iqbal, Paul N. Bennett, Kori Inkpen, Jaime Teevan, Ruth Kikin-Gil, and Eric Horvitz. 2019. Guidelines for HumanAI Interaction. In Proceedings of the 2019 CHI Conference on Human Factors in Computing Systems. ACM.

[3] ARM | Northstar. 2017. AI Today, AI Tomorrow. Awareness and Anticipation of AI: A Global Perspective. https://www.arm.com/solutions/artificial-intelligence/ survey/

[4] Chelsea Barabas, Madars Virza, Karthik Dinakar, Joichi Ito, and Jonathan Zittrain. 2018. Interventions over predictions: Reframing the ethical debate for actuarial risk assessment. In Proceedings of the Conference on Fairness, Accountability, and Transparency. 62-76.

[5] Hugh Beyer and Karen Holtzblatt. 1997. Contextual Design: Defining Customercentered Systems. Elsevier.

[6] Paul P. Biemer and Sharon L. Christ. 2008. Weighting Survey Data. In International Handbook of Survey Methodology, Edith D. de Leeuw, Joop J. Hox, and Don A. Dillman (Eds.). Lawrence Erlbaum Associates New York, NY, 317-341.

[7] Blumberg Capital. 2019. Artificial Intelligence in 2019: Getting Past the Adoption Tipping Point.

[8] Erik Brynjolfsson and Andrew McAfee. 2014. The Second Machine Age: Work, Progress, and Prosperity in a Time of Brilliant Technologies. WW Norton \& Company.

[9] Taina Bucher. 2017. The algorithmic imaginary: exploring the ordinary affects of Facebook algorithms. Information, Communication \& Society 20, 1 (2017), 30-44.

[10] Joy Buolamwini and Timnit Gebru. 2018. Gender shades: Intersectional accuracy disparities in commercial gender classification. In Proceedings of the Conference on Fairness, Accountability, and Transparency. 77-91.

[11] Michelle Carney. 2019. Machine Learning and User Experience: A Few Resources. Medium (9 September 2019)

[12] Daniel Castro. 2019. The U.S. May Lose the AI Race Because of an Unchecked Techno-Panic. Center for Data Innovation (March 2019).

[13] Stephen Cave, Kate Coughlan, and Kanta Dihal. 2019. "Scary Robots": Examining Public Responses to AI. In Proceedings of the 2019 AAAI/ACM Conference on AI, Ethics, and Society (AIES 2019). 331-337.

[14] Stephen Cave, Claire Craig, Kanta Sarasvati Dihal, Sarah Dillon, Jessica Montgomery, Beth Singler, and Lindsay Taylor. 2018. Portrayals and perceptions of AI and why they matter. The Royal Society.

[15] CBS News. 2016. 60 Minutes/Vanity Fair Poll: Artificial Intelligence.

[16] Stevie Chancellor, Michael L. Birnbaum, Eric D. Caine, Vincent M. B. Silenzio, and Munmun De Choudhury. 2019. A Taxonomy of Ethical Tensions in Inferring Mental Health States from Social Media. In Proceedings of the Conference on Fairness, Accountability, and Transparency. 79-88. https://doi.org/10.1145/3287560.3287587

[17] Michael A. DeVito, Jeremy Birnholtz, and Jeffery T. Hancock. 2017. Platforms, people, and perception: Using affordances to understand self-presentation on social media. In Proceedings of the 2017 ACM Conference on Computer Supported Cooperative Work and Social Computing. ACM, 740-754.

[18] Thomas G. Dietterich and Eric Horvitz. 2015. Rise of concerns about AI: Reflections and directions. Commun. ACM 58, 10 (2015), 38-40.

[19] DuckerFrontier. 2019. Smart building trends in 2019: Part 1.

[20] Tim Dutton. 2018. An Overview of National AI Strategies. Medium (28 June 2018).

[21] Edelman. 2019. 2019 Edelman AI Survey.

[22] Motahhare Eslami, Aimee Rickman, Kristen Vaccaro, Amirhossein Aleyasen, Andy Vuong, Karrie Karahalios, Kevin Hamilton, and Christian Sandvig. 2015. "I always assumed that I wasn't really that close to [her]": Reasoning about Invisible Algorithms in News Feeds. In Proceedings of the 33rd Annual ACM Conference on Human Factors in Computing Systems. ACM, 153-162.

[23] Ethan Fast and Eric Horvitz. 2017. Long-term trends in the public perception of artificial intelligence. In Thirty-First AAAI Conference on Artificial Intelligence.

[24] Jessica Fjeld, Nele Achten, Hannah Hilligoss, Adam Nagy, and Madhulika Srikumar. 2020. Principled artificial intelligence: Mapping consensus in ethical and rights-based approaches to principles for AI. Berkman Klein Center Research Publication (2020).

[25] Cary Funk, Alec Tyson, Brian Kennedy, and Courtney Johnson. 2020. Science and Scientists Held in High Esteem Across Global Publics. Pew Research Center (September 2020).

[26] Colin Garvey and Chandler Maskal. 2019. Sentiment Analysis of the News Media on Artificial Intelligence Does Not Support Claims of Negative Bias Against Artificial Intelligence. OMICS: a fournal of Integrative Biology (2019).

[27] Stephen Hawking, Max Tegmark, and Frank Wilczek. 2014. Transcendence looks at the implications of artificial intelligence - but are we taking AI seriously enough? The Independent (May 2014).

[28] High-Level Expert Group on Artificial Intelligence. 2019. Ethics Guidelines for Trustworthy AI.

[29] Timothy R. Hinkin. 1998. A brief tutorial on the development of measures for use in survey questionnaires. Organizational Research Methods 1, 1 (1998), 104-121.

[30] Allyson L. Holbrook, Melanie C. Green, and Jon A. Krosnick. 2003. Telephone versus face-to-face interviewing of national probability samples with long questionnaires: Comparisons of respondent satisficing and social desirability response bias. Public Opinion Quarterly 67, 1 (2003), 79-125.

[31] Christian Homburg, Nicole Koschate, and Wayne D. Hoyer. 2006. The Role of Cognition and Affect in the Formation of Customer Satisfaction: A Dynamic Perspective. Journal of Marketing 70, 3 (2006), 21-31. https://doi.org/10.1509/ jmkg.70.3.021 
[32] Eric Horvitz and Bart Selman. 2012. Interim Report from the Panel Chairs: AAAI Presidential Panel on Long-Term AI Futures. In Singularity Hypotheses. Springer, 301-308.

[33] International Federation of Robotics. 2018. Robot density rises globally.

[34] Ipsos. 2019. Widespread concern about artificial intelligence. https://www.ipsos. com/en-vn/widespread-concern-about-artificial-intelligence/

[35] Anna Jobin, Marcello Ienca, and Effy Vayena. 2019. The global landscape of AI ethics guidelines. Nature Machine Intelligence 1 (September 2019), 389-399.

[36] Shivaram Kalyanakrishnan, Rahul Alex Panicker, Sarayu Natarajan, and Shreya Rao. 2018. Opportunities and Challenges for Artificial Intelligence in India. In Proceedings of the 2018 AAAI/ACM Conference on AI, Ethics, and Society. ACM, 164-170.

[37] Ingrid Y. Lin. 2004. Evaluating a servicescape: the effect of cognition and emotion International fournal of Hospitality Management 23, 2 (2004), 163-178. https: //doi.org/10.1016/j.ijhm.2003.01.001

[38] Lloyd's Register Foundation. 2020. World Risk Poll Report 2019.

[39] Josh Lovejoy and Jess Holbrook. 2017. Human-Centered Machine Learning: 7 steps to stay focused on the user when designing with ML. Medium (9 July 2017).

[40] Pamela McCorduck. 1979. Machines Who Think. A K Peters/CRC Press.

[41] Nora McDonald, Sarita Schoenebeck, and Andrea Forte. 2019. Reliability and InterRater Reliability in Qualitative Research: Norms and Guidelines for CSCW and HCI Practice. In Proceedings of the 22nd ACM Conference on Computer Supported Cooperative Work and Social Computing (CSCW 2019). https://doi.org/10.1145/ 3359174

[42] Luana Micallef and Peter Rodgers. 2014. eulerAPE: drawing area-proportional 3-Venn diagrams using ellipses. PloS one 9, 7 (2014), e101717.

[43] Mozilla. 2019. We Asked People Around the World How They Feel About Artificial Intelligence. Here's What We Learned. https: //foundation.mozilla.org/en/blog/we-asked-people-around-the-world-howthey-feel-about-artificial-intelligence-heres-what-we-learned/

[44] Nature. 2019. AI and big data healthcare in Korea. Nature Focal Point (March 2019).

[45] Lisa-Maria Neudert, Aleksi Knuutila, and Philip N. Howard. 2020. Global Attitudes Towards AI, Machine Learning \& Automated Decision Making: Implications for Involving Artificial Intelligence in Public Service and Good Governance. Oxford Internet Institute (2020).

[46] Nils J. Nilsson. 2009. The Quest for Artificial Intelligence: A History of Ideas and Achievements. Cambridge University Press.

[47] Northeastern University and Gallup. 2018. Optimism and Anxiety: Views on the Impact of Artificial Intelligence and Higher Education's Response.
[48] OpenAI. 2019. Better Language Models and Their Implications. OpenAI Blog (Feb 2019).

[49] Emilee Rader and Rebecca Gray. 2015. Understanding user beliefs about algorithmic curation in the Facebook news feed. In Proceedings of the 33rd Annual ACM Conference on Human Factors in Computing Systems. ACM, 173-182.

[50] Manish Raghavan, Solon Barocas, Jon Kleinberg, and Karen Levy. 2020. Mitigating Bias in Algorithmic Hiring: Evaluating Claims and Practices. In Proceedings of the 2020 Conference on Fairness, Accountability, and Transparency. 469-481.

[51] Nithya Sambasivan and Jess Holbrook. 2019. Toward responsible AI for the next billion users. Interactions 26, 1 (2019), 68-71.

[52] Javier Sánchez-Monedero, Lina Dencik, and Lilian Edwards. 2020. What does it mean to 'solve' the problem of discrimination in hiring?: Social, technical and legal perspectives from the UK on automated hiring systems. In Proceedings of the 2020 Conference on Fairness, Accountability, and Transparency. 458-468. https://doi.org/10.1145/3351095.3372849

[53] Paul Scharre. 2018. Army of none: Autonomous weapons and the future of war. WW Norton \& Company.

[54] Peter Stone, Rodney Brooks, Erik Brynjolfsson, Ryan Calo, Oren Etzioni, Greg Hager, Julia Hirschberg, Shivaram Kalyanakrishnan, Ece Kamar, Sarit Kraus, et al. 2016. Artificial intelligence and life in 2030. One Hundred Year Study on Artificial Intelligence: Report of the 2015-2016 Study Panel (2016), 52.

[55] The European Commission. 2017. Special Eurobarometer 460: Attitudes towards the impact of digitisation and automation on daily life.

[56] United Nations. 2014. World Economic Situation and Prospects: Country classification. https://www.un.org/en/development/desa/policy/wesp/wesp_current/ 2014wesp_country_classification.pdf

[57] Blase Ur, Pedro Giovanni Leon, Lorrie Faith Cranor, Richard Shay, and Yang Wang. 2012. Smart, useful, scary, creepy: perceptions of online behavioral advertising. In Proceedings of the Eighth Symposium on Usable Privacy and Security (SOUPS) 2012.

[58] Jeffrey Warshaw, Nina Taft, and Allison Woodruff. 2016. Intuitions, Analytics, and Killing Ants: Inference Literacy of High School-educated Adults in the US. In Proceedings of the Twelfth Symposium on Usable Privacy and Security (SOUPS) 2016. 271-285.

[59] Darrell M. West. 2018. Brookings survey finds divided views on artificial intelligence for warfare, but support rises if adversaries are developing it. Brookings (August 2018)

[60] Jia You. 2015. A 100-year study of artificial intelligence? Microsoft Research's Eric Horvitz explains. Science (January 2015).

[61] Baobao Zhang and Allan Dafoe. 2019. Artificial intelligence: American attitudes and trends. Available at SSRN 3312874 (2019). 